\title{
A DISCUSSION ABOUT THE EFFECTIVENESS OF SENSORY INTEGRATION IN THE TREATMENT OF ADULTS WITH AUTISTIC SPECTRUM DISORDER
}

\author{
Ginny Moore, Senior Occupational Therapist \\ Adult Learning Disabilities Community Health Team, Kendal
}

\begin{abstract}
This paper was written as the author's course assignment for 'Principles and practice in working with individuals with autistic spectrum disorder'St Martin's College, 2002.
\end{abstract}

The rationale behind the choice of this subject is to look at the effectiveness of sensory integration as a treatment method for adults with autistic spectrum disorder. An occupational therapist (OT) working with adults with a learning disability has many clients referred who are on the autistic spectrum as well as having other difficulties which often are the result of physical problems. Clients are often referred because they have sensory problems which impact on their daily functioning and/or have behavioural problems which can often be attributed to sensory problems. Sensory difficulties in autism are often overlooked.

The practice model of sensory integration is probably the most extensively researched and developed model of practice in occupational therapy. It was originally developed for work with children. Therapists have begun to apply the knowledge of sensory integrative development and the principals of sensory integrative therapy to other client groups such as individuals with autistic spectrum disorders and learning disabilities. Therapists with sensory integration training can diagnose how an individual's sensory systems are working and then design an environment that enables them to interact more effectively.

Children and adults with autism, as well as those with other developmental disabilities, frequently have dysfunctional sensory systems and are called sensory defensive. In some cases sensory dysfunction may be the focus for diagnostic investigation. Sometimes one or more senses are either over- or under-reactive to stimulation. Such sensory problems may be the underlying reasons for such behaviours as rocking, spinning, and hand flapping. This will be discussed later on.

People with autism are often very sensitive to particular stimuli and events in their environment. Stimuli can be sensory experiences in any modality - sight, sound, touch, taste or smell. Sensitivity may be expressed as heightened awareness - the person picks up sounds that others cannot hear, or sees particles of dust or dirt that others do not notice. It may be expressed as a reduced tolerance of particular stimuli - particular colours or patterns, anything which breaks up the smooth line of a wall. Such reduced tolerance may spill over into aversive reaction when the sensory stimuli create physical distress - certain sounds are experienced as painful, certain types of light as uncomfortable, certain textures as intolerable.
People on the autistic spectrum often have a disturbance of sensory modulation which could be under-reactive or overreactive to sensory stimuli. Modulation is when one regulates, adjusts or adapts to vary the pitch, intensity or frequency of incoming information. Sensory integrative procedures have been found to be most effective with people who overreact to sensory stimuli.

The links between sensitivities and behaviour are likely to be direct, although not always easily recognised by others. The distress caused by the sensitivity may be so extreme that it leads to loss of behavioural control. Alternatively, the distress may cause the person to take control by initiating behaviours that will terminate the stimulus directly or get them out of the intolerable situation. Constantly working against the sensory system will eventually result in increased behavioural difficulties.

In general self-stimulating behaviours are attempts at calming. The autistic person's calming methods may seem bizarre, but they usually involve biting, chewing or sucking, or rhythmic rocking.

If we know what concerns a person, the thing to which they are sensitive, or worry about, we can often support them in a way that reduces the anxiety and prevents loss of control. Problems can arise if we are unaware of the sensitivity.

The best way to manage sensitivities is to provide reassurance and avoid unnecessary exposure to the sensitivity and then work towards building a tolerance of the sources of disturbance. A sensory integration assessment can help identify the sensitivities by looking at behaviours.

People with autism often process information very slowly and they must be given time to respond. Non-verbal adults will process sensory input more slowly than verbal adults. Some individuals with very severe sensory processing problems may take several hours to recover after sensory overload.

Lorna Jean King, one of the pioneers of sensory integration, says 'Based on our experience which has been pretty extensive, I would say that 85 to $90 \%$ of autistic children have sensory integration problems. Some are more obvious than others. A therapist's trained eye may pick out subtle signs which may be quite significant, but which a parent might not realise were important. Often small gains in helping the child be less sensitive to sensory input can make big changes in behaviour.' 
Sensory integration therapy is a sensory motor treatment based upon theories developed over the last 30 years by Dr A Jean Ayres. Ayres developed sensory integration theory to explain the relationship between behaviour and neural functioning in order to determine the optimal mode of treatment $^{(2)}$

\section{Sensory integration has three major postulates:}

1 That normal individuals take in sensory information derived from the environment and from movement of their bodies, process and integrate these sensory inputs within the central nervous system, and use this information to plan and organise behaviour

2 Deficits in integrating sensory inputs result in deficits in conceptual and motor learning.

3 The provision of enhanced sensory experiences, provided within the context of a meaningful activity and the planning and production of an adaptive behaviour, results in enhanced sensory integration, and, in turn, enhanced learning.

Some assumptions underlie each of these three postulates:

- Neural plasticity: the brain structure is able to change or be modified

- Sensory integrative process occurs in a developmental sequence

- The brain functions as an integrated whole, but comprises systems that are hierarchically organised

- Evincing an adaptive behaviour promotes sensory integration, and, in turn, the ability to produce an adaptive behaviour reflects sensory integration

- People have an inner drive to develop sensory integration through participation in sensorimotor activities.

The goal of sensory integrative therapy is to facilitate the development of the nervous system's ability to process sensory input in a more normal way. Sensory integration is a term for a process in the normal brain which pulls together all of the various sensory messages in order to form coherent information on which we can act. Basically everything we do requires sensory integration. This normal process can be missing or very badly organised in some people, notably autistic individuals

Sensory integration works with all the senses, but the vestibular, tactile and proprioceptive senses are important because they are such large sources of input.

The vestibular system provides the brain with feedback regarding body motion and position. It is located in the inner ear and relates us to gravity. It gives us our sensation of the weight of our body. It also tells us where we are in space, standing up, or on our head; whether we are falling, or turning our head. It monitors our head and body movements in any direction. It works 24 hours a day, and therefore is a very big source of input.

The prevalence of toe walking among people with autism has been associated with extreme sensitivity in the feet. In children it is often associated with neurological immaturity, but another explanation could be a dysfunctional vestibular system. It may be possible to reduce or eliminate toe walking by providing the person with therapeutic vestibular stimulation (eg being swung on a glider swing).
The proprioceptors are the neuroceptors in tendons, muscles and joints. They tell us where our foot is when we pull it back to kick a ball, or how high our hand is when we reach up to comb our hair. Because the proprioceptors are getting input whenever we move, they are a large source of sensory input. Proprioceptive input can vary in intensity. When one jumps on a trampoline, there is more intense input to the ankles, knees and hips than there is in walking.

The tactile system has three different types of receptors. One responds to light touch. This is a protective, alerting sense which makes us check on what is touching us in case it might be dangerous. The second receptor is discriminative touch, eg when you reach in a pocket you know by feel what you touch. The third set of touch receptors is those that receive information about heat, cold and pain.

People who do not process sensory information efficiently often do not have good reception of heat cold and pain input. As sensory integration becomes more efficient through therapy, they often become more normally aware of heat, cold and pain. Sensory integration therapy seeks to help the person be less hypersensitive to light touch, better able to use discriminative touch to learn, more able to respond to heat, cold, and pain, and thus be more safe from harm.

Temple Grandin ${ }^{(3)}$ craved deep pressure stimulation and thus constructed her squeeze machine to satisfy her craving for the feeling of being held. The machine was designed so that she could control the amount and duration of the pressure. Grandin describes the over-sensitivity of her nervous system slowly reducing and a stimulus that was once aversive and overwhelming becoming pleasurable. Using the squeeze machine enabled her to tolerate another person touching her.

Several squeeze machines are now being used at sensory integration clinics in the United States. Therapists have found that some hyperactive and autistic children will immediately use the machine, and others are so oversensitive to touch that they initially avoid the machine and other activities involving touch. Activities involving touch become pleasurable when the nervous system becomes desensitised (a modulation difficulty), eg people who cannot tolerate tooth-brushing could be desensitised through gently rubbing them around the mouth.

The interconnections of these three basic senses (vestibular, tactile and proprioceptive) start forming before birth and continue to develop as the person matures and interacts with his/her environment. The three senses are not only interconnected but are also connected with other systems in the brain. Although these three sensory systems are less familiar than vision and audition, they are critical to our basic survival. The inter-relationship among these three senses is complex. Basically, they allow us to experience, interpret and respond to different stimuli in our environment.

When working with people with autism other perspectives such as that of Donna Williams must be taken into account ${ }^{(4)}$. She describes some people with autism as working on 'mono', ie that some people with autism only use their eyes or ears or their sense of touch or smell at any one time, not simultaneously, eg a person may look away when listening to something or they may use their sense of touch rather than their eyes to orient them or identify things. Sensory integration seeks to get the person to use several senses at once. But we must ask why sensory integration has not taken place naturally in the first place. This could be because the 
brain is not able to filter out enough information on each sensory channel for it to be able to cope efficiently with all the information at once. Therefore the therapist has to be careful how the therapy is administered so that a person does not merely 'switch channels' when two channels are required or information overload takes place, causing an attack of sensory hypersensitivity (which can result in behavioural problems). Or it could result in 'total systems shutdown' and avoidance.

Despite most of the research on sensory integration involving children, $\mathrm{King}^{(1)}$ believes that a person is never too old to benefit from sensory integrative therapy, since we know that the nervous system develops by making new connections between neurons throughout life. As some connections disappear through disuse, other connections can be formed as a result of new experience. So the brain is adaptable at any age.

When a client is referred for sensory integration treatment there is a period of assessment. This uses observations and videoing plus the gathering of information from people who know the client well. Behaviour, likes and dislikes are the main focus of the assessment. The results are then analysed using the three sensory domains and divided into sensory modulation disorders (hypo-reactive and hyper-reactive), sensory discrimination disorders, (postural tone/control, bilateral integration, tactile discrimination and visual/spatial) and problems with body scheme and praxis, neuro-motor problems and behavioural traits. If an area of difficulty is shown, a suitable programme of intervention can be planned, eg Calming vestibular activities such as swinging side to side using a hammock or using a rocking chair. These activities can initially be part of the therapist's treatment programme and then be incorporated into the person's everyday activities, eg carrying heavy shopping bags to increase proprioceptive feedback or sleeping under heavy blankets to facilitate deep touch/ pressure, which can be very calming.

Although autism was formerly thought to be a psychiatric disturbance it is now recognised to be associated with neurological impairment. Developmental abnormalities of cell structure have been found in both the cerebellum and limbic regions of the brain. The limbic system is a complex set of structures with many associated functions including self preservation, and given its hypothesised role in sensory modulation and sensory defensiveness disorders, it is not surprising that this is one area of the brain in which abnormalities have been found in autism.

Waterhouse, Fein and Modah ${ }^{(5)}$ looked at neurofunctional mechanisms in autism. Behavioural impairments in autism are thought to result from abnormal neuronal organisation in brain development, generating four systemically neurofunctional impairments, one of which is canalesthesia, wherein abnormal hippocampal system function 'canalizes' sensory records, disrupting the integration of information.

Autopsies of nine autistic brains revealed abnormalities in the cerebellum, hippocampus, amygdala and other parts of the limbic system ${ }^{(6)}$. EEG studies indicated that autistic children have severe abnormalities in their capacity to shift attention between visual and auditory stimuli. Brain structures that control attention shift are connected to the cerebellar vermis. Abnormalities in attention-shifting may be the basis of perseverate behaviour and some social deficits. This may explain why treatments that stimulate the cerebellum, and certain sensory treatments, often improve overall behaviour.
Literature and papers were reviewed to look at how sensory integration has been used to treat people with autism. Unfortunately the limited research that has been done relates to children rather than adults. This shows a need for good research to be carried out in this area. Summaries of findings are discussed:

Donna Williams ${ }^{(4)}$ reports that sensory integration treatment, consisting of rubbing her skin with brushes, has helped. Even though she disliked the tactile input from the brushes, she reported that it helped her different sensory systems to work together and become more integrated. Her sensory processing also becomes more normal when she is relaxed and is focusing on only one sensory channel (working on 'mono').

Watling, Deitz, Kanny, and McLaughlin( ${ }^{(7)}$ studied the current practice patterns of occupational therapists experienced in working with children with autistic spectrum disorders was looked at. A questionnaire was used to look at practice patterns, theoretical approaches, intervention techniques, and preferred methods for work with children with autism. Results revealed that intervention services were typically provided in a one-to-one format with the most common techniques being sensory integration (99\%) and positive reinforcement (93\%). Theoretical approaches included sensory integration (99\%), developmental (88\%), and behavioural $(73 \%)$.

Other studies have been done looking at the use of sensory integration. Case-Smith and Bryan ${ }^{(8)}$ looked at the effects of an occupational therapy intervention emphasising sensory integration with five pre-school children with autism. The results showed that when the baseline and intervention phases of the study were compared, four children demonstrated decreased frequency of non-engaged behaviour, and three demonstrated increased frequency of mastery (goaldirected) play. Improvements in the frequency of interactions were minimal. The authors concluded that behavioural changes can be made with children with autism when participating in intervention using a sensory integration approach.

Watling ${ }^{(9)}$ looked at selected literature exploring the effectiveness of a sensory-based approach to the treatment of autism. All of the literature appears to concern children rather than adults. Watling concludes that few of the available articles provide sufficient data for or against the effectiveness of sensory-based intervention with this challenging population and that further research is needed in this area using well-designed studies with appropriate sample sizes and controls.

Dawson and Watling ${ }^{(10)}$ looked at interventions to facilitate auditory, visual and motor integration in autism. They looked at the three interventions designed to address such abnormalities: sensory integration therapy, traditional occupational therapy and auditory integration. Although sensory processing and motor abnormalities are neither universal nor specific to autism, the prevalence of such abnormalities in autism is relatively high. There is, however, little controlled research on the effectiveness of interventions designed to address these abnormalities. Four objective outcome studies of sensory integration therapy were identified. These were of such small scale that no firm conclusions regarding efficacy could be made. 


\section{CONCLUSION}

To conclude, research studies carried out so far do not indicate that sensory integration has any specific effect, although it is not known to cause any harm. It is a widelyused approach, especially by therapists in the United States.

While sensory integrative procedures may be appropriate for those who display problems with modulation of sensory inputs, we must remember that autism is associated with brain abnormalities, not merely with dysfunction. Care is required not to over step the boundaries of sensory integration theory when applying these procedures to children and adults with autism. However, sensory integration theory can assist us in understanding the effective reactions to sensory inputs often seen in these clients. Therefore one concludes that a sensory integration approach is a valid intervention for occupational therapists to use when working with adults with autistic spectrum disorder. Hopefully in future further research will support this.

\section{REFERENCES}

1 King LJ. Stephen M Edelson. Interview with Lorna Jean King 1996 http://www.autism.org/interview/ljk.html

2 Fisher AG, Murray EA, Bundy A. Sensory Integration Theory and Practice. FA Davies Co, Philadelphia 1991
3 Grandin T. My experiences with visual thinking, sensory problems and communication difficulties/

http://www.autism.org/temple/visual.html

4 Williams D. Autism; an inside out approach. Jessica Kinsley, London and Bristol, Pennsylvania. 1999 pp108-109

5 Waterhouse L, Fein D, Modahl C. Neurofunctional mechanisms in autism. Psychol Rev 1996;103(3)457-89

6 Bauman M, Kemper T. Neuroanatomic observations of the brain in autism. In Bauman and Kemper The neurobiology of autism. J Hopkins Univ Press, Baltimore 1994. pp119-145

7 Watling R, Deitz J, Kanny EM, McLaughlin JF. Current practice of occupational therapy for children with autism. Am J Occ Therap 1999;53(5)498-505

8 Case-Smith J, Bryan T. The effects of occupational therapy with sensory integration emphasis on pre-schoolage children with autism. Am J Occ Ther 1999;53(5)489-97

9 Watling R. Selected literature exploring the effectiveness of a sensory-based approach to the treatment of autism. Phys \& Occ Ther in Paeds 1998;18(2)77-85

10 Dawson G, Watling R. Interventions to facilitate auditory, visual and motor integration in autism; a review of the evidence. J Autism \& Develop Disorders 2000;30(5)415-21 